\title{
Avaliação físico-química e comportamento higroscópico de goiaba em pó obtida por spray-dryer ${ }^{1}$
}

\author{
Physicochemical evaluation and hygroscopic behavior of powdered guava obtained \\ by spray drying
}

\author{
Alinne Alencar Costa dos Santos ${ }^{2}$, Anne Karine Gurgel Dias Florêncio ${ }^{2}$, Érica Milô de Freitas Felipe Rocha ${ }^{3}$ e \\ José Maria Correia da Costa ${ }^{4 *}$
}

\begin{abstract}
RESUMO - A goiaba é uma das frutas tropicais mais populares e de maior aceitação em todas as regiões do Brasil. Do seu fruto podem-se obter vários produtos alimentícios tais como: doces, geleias, licores e sucos de diversas formas. Diante do exposto, o objetivo desta pesquisa foi caracterizar a polpa de goiaba atomizada quanto à sua composição físicoquímica e, avaliar o seu comportamento higroscópico através das isotermas de adsorção utilizando diferentes modelos matemáticos. As análises físico-químicas realizadas tanto na polpa integral de goiaba quanto no pó de goiaba atomizado foram: umidade, $\mathrm{pH}$, acidez, sólidos solúveis e ácido ascórbico apresentando os seguintes resultados, respectivamente, 88,57 - 5,69\%; 3,76 - 3,88;0,43 - 0,24 mg $100 \mathrm{~g}^{-1} ; 8,43-93,00{ }^{\circ}$ Brix e 2,77 - 3,79 mg $100 \mathrm{~g}^{-1}$. As isotermas de adsorção foram construídas utilizando-se do ajuste de dados experimentais aos modelos matemáticos de GAB, BET, Henderson e Oswin. Sendo, o modelo Henderson, em todas as temperaturas avaliadas, aquele que melhor se ajustaram para o pó de goiaba atomizado com erro variando de 09,93 a 12,09\% e coeficiente de correlação variando de 0,9900 a 0,9934 .
\end{abstract}

Palavras-chave: Goiaba. Frutas. Secagem.

\begin{abstract}
The guava is one of the most popular tropical fruits, being highly accepted all over Brazil. Many food products can be made from the fruit, such as jams, jellies, liquors and many types of juice. Given the above, the objective of this research was to characterise atomised guava pulp as to its physicochemical composition, and assess its hygroscopic behaviour by means of adsorption isotherms employing different mathematical models. The physicochemical analyses, carried out on both the whole guava pulp and on the atomised guava powder, were: moisture; $\mathrm{pH}$; acidity; soluble solids and ascorbic acid, giving the following results respectively: 88.57-5.69\%; 3.76-3.88, 0.43-0.24 mg $100 \mathrm{~g}^{-1} ; 8.43$ to $93.00{ }^{\circ}$ Brix and $2.77-3.79 \mathrm{mg} 100 \mathrm{~g}^{-1}$. The adsorption isotherms were constructed adjusting the experimental data to the mathematical models of GAB, BET, Henderson and Oswin. The Henderson model presented the best fit to the atomised guava powder for all temperatures tested, presenting an error ranging from 09.93 to $12.09 \%$ and a correlation coefficient ranging from 0.9900 to 0.9934 .
\end{abstract}

Key words: Guava. Fruit. Drying.

\footnotetext{
*Autor para correspondência

${ }^{1}$ Recebido para publicação em 16/10/2011; aprovado em 06/01/2014

Trabalho extraído da Pesquisa financiada pelo CNPq

${ }^{2}$ Departamento de Tecnologia de Alimentos, Centro Ciências Agrárias, Universidade Federal do Ceará, Fortaleza-CE, Brasil, linne.acs@ gmail.com, annekarine18@yahoo.com.br

${ }^{3}$ Instituto Federal de Educação, Ciência e Tecnologia do Rio Grande do Norte/IFRN, Campus Pau dos Ferros, Pau dos Ferros-RN, Brasil, emffrocha@yahoo.com.br

${ }^{4}$ Departamento de Tecnologia de Alimentos, Universidade Federal do Ceará, Bloco 858, Campus do Pici, Av. Mister Hull 2977, Alagadiço, Fortaleza-CE, Brasil, 60.356-000, correia@ufc.br
} 


\section{INTRODUÇÃO}

A goiaba (Psidium guajava) é um fruto tropical, climatérico e apresenta altas taxas de transpiração e perda de massa após sua colheita (AZZOLINI et al., 2005). Devido ao intenso metabolismo durante o amadurecimento, esses frutos senescem rapidamente, impedindo o armazenamento por períodos prolongados (CARVALHO et al., 2001; RIBEIRO et al., 2005; VILA et al., 2007). De acordo com os dados do Instituto Brasileiro de Geografia e Estatística (2011), o Brasil no ano de 2009 produziu 297.377 toneladas de goiaba, sendo a região Nordeste responsável por $46,35 \%$ desta produção.

Como a comercialização de frutos está restrita a sua época de safra e, devido a sua alta perecibilidade, é importante, para viabilizar as diversas agroindústrias, de unidades de processamento que possam alimentar as indústrias fora do período de safra. Um dos processos utilizados nos últimos anos para prolongar a vida útil dos frutos é a secagem por atomização. Este processo tem por finalidade transformar a polpa de fruta em forma de pó, permitindo um armazenamento prolongado e uma maior estabilidade e longevidade do produto, de modo a ter uma maior vida de prateleira, podendo o consumidor ter acesso a esse produto durante o ano, e não apenas no período de safra (ANSELMO et al., 2006).

A secagem por spray-dryer ou atomização é um processo amplamente utilizado na indústria de alimentos, e em condições ideais de processamento, tem se mostrado eficaz na obtenção de diversos produtos. Este processo de desidratação, quando utilizado em alimentos ricos em açúcar tais como sucos de frutas, mel e derivados do amido altamente hidrolisado, apresenta grande potencial econômico e, a transformação destes produtos em forma de pós alimentícios desidratadas resulta em alimentos de volume reduzido, com uma longa vida útil e uma boa reconstituição (ADHIKARI et al., 2004).

No entanto, os sucos de fruta obtidos por spray-dryer apresentam obstáculos em suas propriedades funcionais, com alta pegajosidade (stickiness) e higroscopicidade, que tornam a sua embalagem e utilização substancialmente mais difíceis (CANO-CHAUCA et al., 2005).

Diante do exposto, este trabalho teve como principais objetivos: avaliar as características físicoquímicas do pó de goiaba atomizado e verificar o seu comportamento higroscópico através das isotermas de adsorção utilizando diferentes modelos matemáticos.

\section{MATERIAL E MÉTODOS}

As polpas de goiaba selecionadas para a realização deste trabalho foram adquiridas no comercio varejista de Fortaleza - CE e, então conduzidas ao Laboratório de Controle de Qualidade e Secagem do Departamento de Tecnologia de Alimentos da Universidade Federal do Ceará, onde foram mantidas sob congelamento em um freezer vertical, a $-18{ }^{\circ} \mathrm{C}$, sendo o seu descongelamento realizado na embalagem original do produto, em geladeira $\left(2{ }^{\circ} \mathrm{C}\right)$ por 18 horas.

Após descongelamento, elaborou-se uma polpa formulada composta de polpaintegral de goiabaadicionada de $10 \%$ de maltodextrina e $50 \%$ de água destilada (p/p). Após completa dissolução e homogeneização, a solução formulada foi então submetida à secagem em um Spraydryer modelo MSD 1.0 da marca Labmaq do Brasil, utilizando um bico pneumático de 1,2 mm, vazão de bombeamento da polpa de $500 \mathrm{~L} \mathrm{~h}^{-1}$, vazão de ar quente de $3,5 \mathrm{~L} \mathrm{~h}^{-1}$ e temperatura de entrada de $120^{\circ} \mathrm{C}$.

Para todas as determinações físico-químicas, tanto da polpa integral quanto do pó atomizado obtido, foram realizadas em triplicata, sendo elas: umidade (determinada utilizando uma estufa a vácuo a $70^{\circ} \mathrm{C}$, sob pressão reduzida, até peso constante), $\mathrm{pH}$ (determinado através de um medidor de $\mathrm{pH}$, calibrado periodicamente com soluções tampão de pH 4,0 e 7,0), acidez titulável (determinada por titulação com $\mathrm{NaOH} 0,1 \mathrm{~N}$ e os resultados expressos em percentagem de ácido cítrico) e, os sólidos solúveis (determinados através da leitura dos ${ }^{\circ}$ Brix a $20^{\circ} \mathrm{C}$ em refratômetro, com escala variando de 0 a $90{ }^{\circ}$ Brix) e, a análise de açucares totais e redutores foram realizadas segundo a metodologia do Instituto Adolfo Lutz (2004). O conteúdo de ácido ascórbico foi determinado através de método titulométrico baseado na redução do indicador 2,6-diclorofenolindofenol pelo ácido ascórbico, sendo os resultados expressos em mg de ácido ascórbico por $100 \mathrm{~g}$ de amostra, segundo a metodologia descrita por Strohecker e Henning (1967). A atividade de água do pó foi feita utilizando um medidor de atividade de água, modelo AQUALab (1997) 4TEV nas temperaturas de $25 ; 30 ; 35$ e $40{ }^{\circ} \mathrm{C}$, conforme indicações do fabricante.

Para as determinações das isotermas de adsorção, a amostra foi pesada, em triplicata, com massas de aproximadamente $0,20 \mathrm{~g}$ de cada e colocada em cadinhos de alumínio, previamente tarados. Posteriormente, os cadinhos foram colocados sobre suporte contido dentro das células isoladas, as quais continham as soluções salinas saturadas de: $\mathrm{CH}_{3} \mathrm{COOK}\left(\mathrm{a}_{\mathrm{w}}=0,21\right) ; \mathrm{K}_{2} \mathrm{CO}_{3}\left(\mathrm{a}_{\mathrm{w}}=0,48\right)$; $\operatorname{NaBr}\left(a_{w}=0,57\right) ; \operatorname{SnCl}_{2}\left(a_{w}=0,76\right) ; \operatorname{KCl}\left(a_{w}=0,84\right)$ e $\mathrm{BaCl}_{2}\left(\mathrm{a}_{\mathrm{w}}=0,90\right)$ de acordo com Greespan (1977). As temperaturas utilizadas para a construção das isotermas foram de $25 ; 30 ; 35$ e $40{ }^{\circ} \mathrm{C}$.

Foram feitas pesagens dos cadinhos, contendo a amostra, a cada 24 horas até atingirem a umidade de equilíbrio 
detectadas através de pesagem numa balança analítica modelo B-TEC-210ª da marca Tecnal. Durante este período a amostra foi submetida à inspeção visual, para detectar quaisquer alterações perceptíveis. Posteriormente, os cadinhos foram então colocados na estufa a $105^{\circ} \mathrm{C}$ para determinação do teor de umidade final de equilíbrio da amostra.

A umidade de equilíbrio (Xeq) foi calculada pela diferença entre a massa que a amostra apresentou no equilíbrio e sua massa seca inicial:

$$
X_{e q}=\frac{M o-M s}{M s}
$$

onde: $\mathrm{X}_{\mathrm{eq}}=$ umidade de equilíbrio ( $\mathrm{g} / \mathrm{g}$ matéria seca); $\mathrm{M}_{\mathrm{o}}=$ massa da amostra no equilíbrio $(\mathrm{g}) ; \mathrm{M}_{\mathrm{s}}=$ massa da amostra seca $(\mathrm{g})$.

Para o ajuste matemático dos dados experimentais das isotermas de adsorção foram utilizados os modelos matemáticos de: BRUNAUER, EMMET e TELLER (BET); GUGGENHEIM-ANDERSON - DE BOER (GAB); HENDERSON; OSWIN, representados, respectivamente, pelas Equações 2, 3, 4 e 5.

$$
\begin{aligned}
& X_{e q}=\frac{X m \cdot C \cdot K \cdot A_{w}}{(1-) K \cdot A_{w} \cdot\left(1-K \cdot A_{w}+C \cdot K \cdot A_{w}\right)} \\
& X_{e q}=\frac{X m \cdot C \cdot A_{w}}{1-A_{w}}\left[\frac{1-(n+1) \cdot\left(A_{w}\right)^{2}+n \cdot\left(A_{w}\right)^{n+1}}{1-(1-C) \cdot A_{w}-C \cdot\left(A_{w}\right)^{n+1}}\right] \\
& X_{e q}=\left[\frac{-\ln \left(1-a_{w}\right)}{b}\right]^{\frac{1}{a}} \\
& X_{e q}=\left[\frac{a_{w}}{1-a_{w}}\right]
\end{aligned}
$$

onde: $\mathrm{a}_{\mathrm{w}}=$ atividade de água; $\mathrm{X}_{\mathrm{m}}=$ conteúdo de umidade na monocamada molecular $\left(\mathrm{g} / \mathrm{g}\right.$ de matéria seca); $\mathrm{X}_{\mathrm{eq}}=$ conteúdo de umidade de equilíbrio (g/g de matéria seca); $\mathrm{C}=$ constante de BET relacionada ao calor de sorção da camada molecular; $a, b$ e $K=$ parâmetros de ajuste.
Os valores do erro (E) apresentados foram calculados de acordo com a Equação 6 (KUROZAWA; EL-AOUAR; MUR, 2005):

$$
E=\frac{100}{n} \sum_{i=l}^{n} \frac{\left|\left(M_{i}-M p_{i}\right)\right|}{M_{i}}
$$

onde: $\mathrm{E}=$ erro médio relativo; $\mathrm{M}_{\mathrm{i}}=$ valores obtidos experimentalmente; $\mathrm{Mp}_{\mathrm{i}}$ = valores preditos pelo modelo; $\mathrm{n}=$ número de dados experimentais.

\section{RESULTADOS E DISCUSSÃO}

Os resultados da caracterização físico-química do pó de goiaba atomizado são expostos na Tabela 1, onde, pode-se observar que os resultados médios de umidade indicam que o pó de goiaba obtido perdeu em média 93,58\% de água em relação à polpa integral. Esse resultado indica que a polpa atomizada está de acordo com os parâmetros estabelecidos pela legislação para produtos desidratados (AGÊNCIA NACIONAL DE VIGILÂNCIA SANITÁRIA, 2005a), sendo este um fator protetor ao desenvolvimento de microorganismos deteriorantes e patogênicos.

$\mathrm{O} \mathrm{pH}$ da polpa integral e do pó foram de, respectivamente, 3,76 e 3,88 . O que indica que esses valores se encontram, do ponto de vista da tecnologia de alimentos, em uma faixa segura em relação ao desenvolvimento microbiano, além de está dentre os valores estabelecidos para o padrão de identidade e qualidade da polpa em questão (BRASIL, 2000). Já para o parâmetro de acidez foi observado um valor para a polpa integral superior ao observado no pó.

O processo de secagem por atomização reduziu acidez titulável da goiaba em pó $\left(0,24 \mathrm{mg} 100 \mathrm{~g}^{-1}\right) \mathrm{em}$ relação ao valor encontrado para a polpa integral de goiaba (0,43 mg $\left.100 \mathrm{~g}^{-1}\right)$; este comportamento era esperado em razão da temperatura ser responsável pelo aumento

Tabela 1 - Caracterização físico-química de polpa de goiaba integral e atomizada

\begin{tabular}{lcc}
\hline \multicolumn{1}{c}{ Análises físico-químicas } & Polpa integral & Pó de goiaba atomizado \\
\hline Umidade (\%) & $88,57 \mathrm{a} \pm 0,07$ & $05,69 \mathrm{~b} \pm 0,28$ \\
$\mathrm{Ph}$ & $3,76 \mathrm{~b} \pm 0,15$ & $03,88 \mathrm{a} \pm 0,05$ \\
Acidez (mg de ácido cítrico $\left.100 \mathrm{~g}^{-1}\right)$ & $0,43 \mathrm{a} \pm 0,00$ & $00,24 \mathrm{~b} \pm 0,05$ \\
Sólidos solúveis $\left({ }^{\circ}\right.$ Brix) a $20^{\circ} \mathrm{C}$ & $8,43 \mathrm{~b} \pm 0,15$ & $93,00 \mathrm{a} \pm 0,26$ \\
Ácido Ascórbico $\left(\mathrm{mg} 100 \mathrm{~g}^{-1}\right)$ & $2,77 \mathrm{~b} \pm 0,29$ & $03,79 \mathrm{a} \pm 0,76$ \\
Açúcares Totais $\left(\mathrm{g} 100 \mathrm{~g}^{-1}\right)$ & $2,84 \mathrm{~b} \pm 0,08$ & $34,64 \mathrm{a} \pm 0,70$ \\
Açúcares Redutores $\left(\mathrm{g} 100 \mathrm{~g}^{-1}\right)$ & $2,79 \mathrm{~b} \pm 0,10$ & $20,25 \mathrm{a} \pm 0,38$ \\
\hline
\end{tabular}

Valores médios, na mesma linha, seguidos de letras minúsculas diferentes apresentam diferença estatisticamente significativa (p $\leq 0,05)$ pelo Teste de Tukey 
do $\mathrm{pH}$ da amostra e, consequentemente, diminuição da acidez. Oliveira, Figueiredo e Queiroz (2006) encontram valores de acidez para pitanga integral e na forma de pó formulada com $15 \%$ de maltodextrina $+30 \%$ de água destilada respectivamente de 2,23 e $1,52 \%$ e, Caleguer e Benassi (2007) em seu estudo com adição de polpa, CMC e fibra em preparados sólidos de laranja observaram teores variando 0,21 a $0,23 \mathrm{mg} 100 \mathrm{~g}^{-1}$.

Tanto por ser um produto mais concentrado devido à retirada de água quanto pela adição de maltodextrina, o pó de goiaba apresentou teor médio de sólidos solúveis e açúcares totais e redutores, significativamente, superior ao observado para polpa integral de goiaba.

Os teores de ácido ascórbico observado tanto para a polpa integral quanto para a atomizada apresentaram diferenças estatística significativas, com nível de $5 \%$ de probabilidade, representando aproximadamente 4,62 e 6,32\% da Ingestão Diária Recomendada (IDR) para adultos (AGÊNCIA NACIONAL DE VIGILÂNCIA SANITÁRIA, 2005b). Comparando-se o teor de ácido ascórbico da polpa integral com o teor obtido para a polpa de goiaba em pó, constata-se que o processo de secagem concentrou este constituinte no pó apresentando um valor de $36,82 \%$ superior.

Os dados experimentais das isotermas de sorção para a polpa de goiaba em pó foram ajustados por diversos modelos da literatura e, os parâmetros estimados por cada modelo estão apresentados na Tabela 2.

De acordo com os valores obtidos dos coeficientes de correlação $\left(\mathrm{R}^{2}\right)$ e dos erros médios $(E)$, pode-se observar que para o pó alimentício obtido da polpa de goiaba atomizada, o modelo de Henderson foi o que melhor se ajustou à curva experimental, em todas as temperaturas analisadas, com os menores valores de erro médio $(E)$ e os maiores valores nos coeficientes de correlação $\left(\mathrm{R}^{2}\right)$. O segundo modelo que melhor representou esses dados experimentais, também em todas as temperaturas avaliadas, foi o de GAB sendo o modelo de BET o que representou o pior ajuste para os dados em questão com $\mathrm{R}^{2}$ variando de 0,9878 a 0,9914 e $E$ variando de 16,84 a $19,31 \%$.

As isotermas de adsorção de polpa de goiaba atomizada foram obtidas traçando os teores de água de

Tabela 2 - Parâmetros de ajuste das isotermas de adsorção aos modelos do pó de polpa de goiaba atomizado, coeficiente de correlação $\left(\mathrm{R}^{2}\right)$ e erro médio (E) na temperatura de $25 ; 30 ; 35$ e $40{ }^{\circ} \mathrm{C}$

\begin{tabular}{|c|c|c|c|c|c|c|}
\hline \multirow{2}{*}{ Modelos } & \multirow{2}{*}{ Temperaturas } & \multicolumn{3}{|c|}{ - Parâmetros----------------- } & \multirow{2}{*}{$\mathrm{R}^{2}$} & \multirow{2}{*}{$\mathrm{E}(\%)$} \\
\hline & & $\mathrm{X}_{\mathrm{m}}$ & $\mathrm{C}$ & $\mathrm{K}$ & & \\
\hline \multirow{4}{*}{ GAB } & 25 & 0,17051 & 0,48719 & 0,85800 & 0,9931 & 11,36 \\
\hline & 30 & 0,10747 & 0,80882 & 0,91514 & 0,9899 & 14,42 \\
\hline & 35 & 0,12456 & 0,73063 & 0,90016 & 0,9899 & 14,36 \\
\hline & 40 & 0,13874 & 0,68971 & 0,89346 & 0,9898 & 14,53 \\
\hline & & $\mathrm{X}_{\mathrm{m}}$ & $\mathrm{C}$ & & & \\
\hline \multirow{5}{*}{ BET } & 25 & 0,20528 & 0,46459 & 1,65871 & 0,9914 & 16,84 \\
\hline & 30 & 0,08375 & 1,51858 & 1,44708 & 0,9878 & 17,17 \\
\hline & 35 & 0,09983 & 0,66513 & 13,22702 & 0,9887 & 19,31 \\
\hline & 40 & 0,09272 & 1,42639 & 1,41056 & 0,9879 & 19,26 \\
\hline & & $\mathrm{a}$ & $\mathrm{b}$ & & & \\
\hline \multirow{5}{*}{ Henderson } & 25 & 0,67473 & 3,81183 & & 0,9934 & 09,93 \\
\hline & 30 & 0,66349 & 3,75433 & & 0,9900 & 11,93 \\
\hline & 35 & 0,67412 & 3,69088 & & 0,9902 & 11,47 \\
\hline & 40 & 0,67593 & 3,55984 & & 0,9900 & 12,09 \\
\hline & & $\mathrm{a}$ & $\mathrm{b}$ & & & \\
\hline \multirow{4}{*}{ Oswin } & 25 & 0,08564 & 0,80915 & & 0,9910 & 17,26 \\
\hline & 30 & 0,08422 & 0,82436 & & 0,9888 & 18,60 \\
\hline & 35 & 0,08838 & 0,82592 & & 0,9887 & 18,30 \\
\hline & 40 & 0,09264 & 0,83903 & & 0,9886 & 18,57 \\
\hline
\end{tabular}


equilíbrio do pó alimentício em função da atividade de água. Os valores experimentais obtidos mostram que o comportamento das isotermas foi do tipo exponencial conforme pode ser verificado nas Figuras $1 ; 2 ; 3$ e 4. $\mathrm{E}$, as curvas obtidas apresentaram formato do tipo III (formato de $\mathrm{J}$ ), característica de alimentos com alto teor de açúcar na classificação da IUPAC conforme Hérbrard et al., (2003). Este tipo de curva também foi observado por Gabas et al. (2007) em polpa de abacaxi adicionada de maltodextrina ou goma arábica e seca a vácuo e, Wang, Zhang e Chen (2008) em gooseberry liofilizada.

Figura 1 - Comportamento das isotermas de adsorção do pó de goiaba para diferentes modelos matemáticos a $25^{\circ} \mathrm{C}$

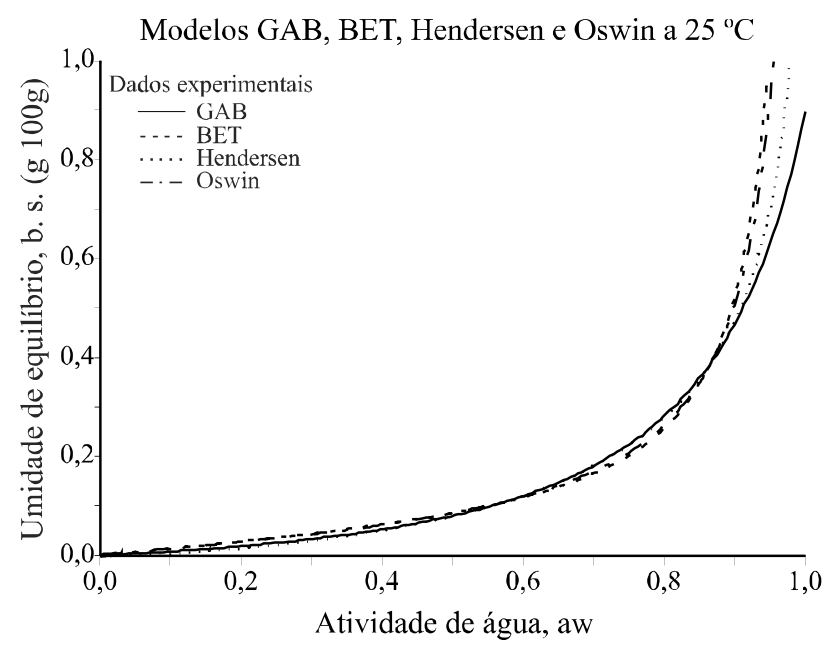

Figura 2 - Comportamento das isotermas de adsorção do pó de goiaba para diferentes modelos matemáticos a $30^{\circ} \mathrm{C}$

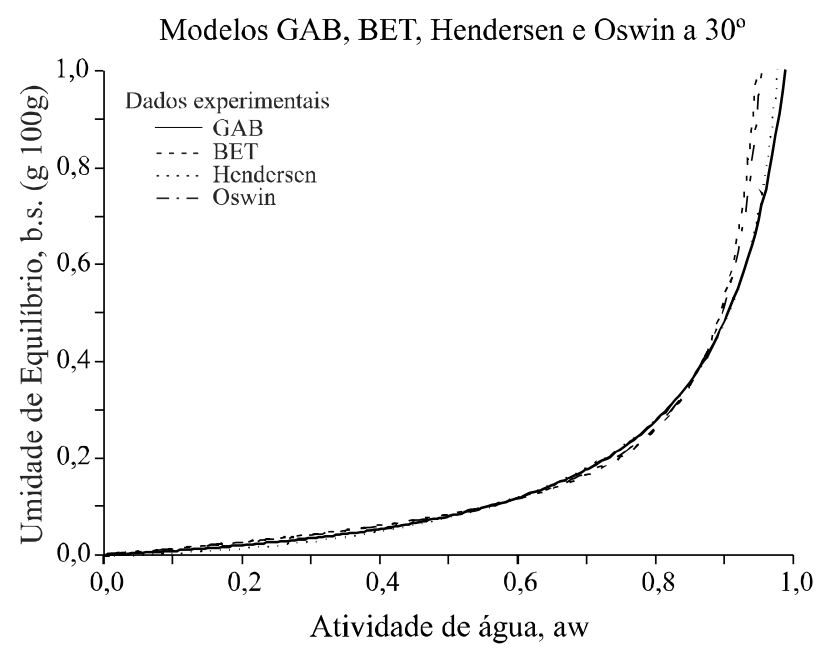

Figura 3 - Comportamento das isotermas de adsorção do pó de goiaba para diferentes modelos matemáticos a $35^{\circ} \mathrm{C}$

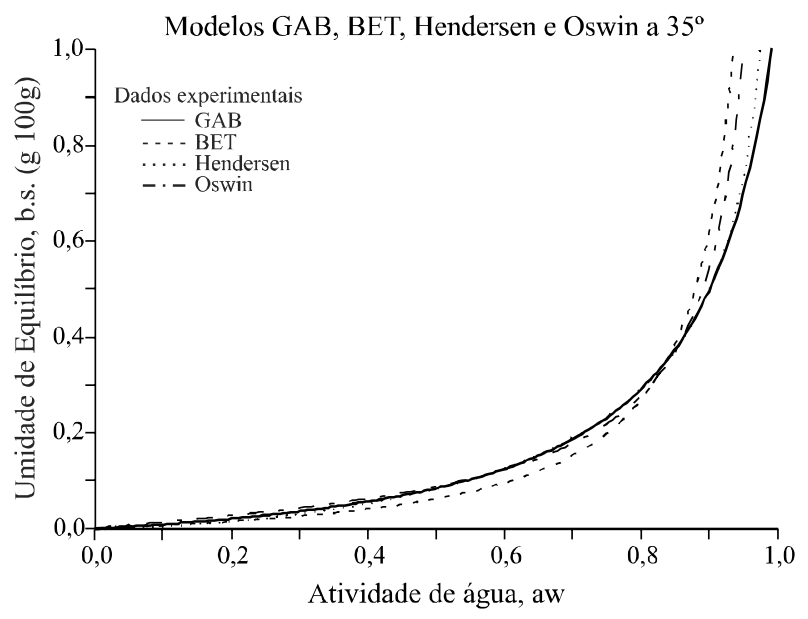

Figura 4 - Comportamento das isotermas de adsorção do pó de goiaba para diferentes modelos matemáticos a $40{ }^{\circ} \mathrm{C}$

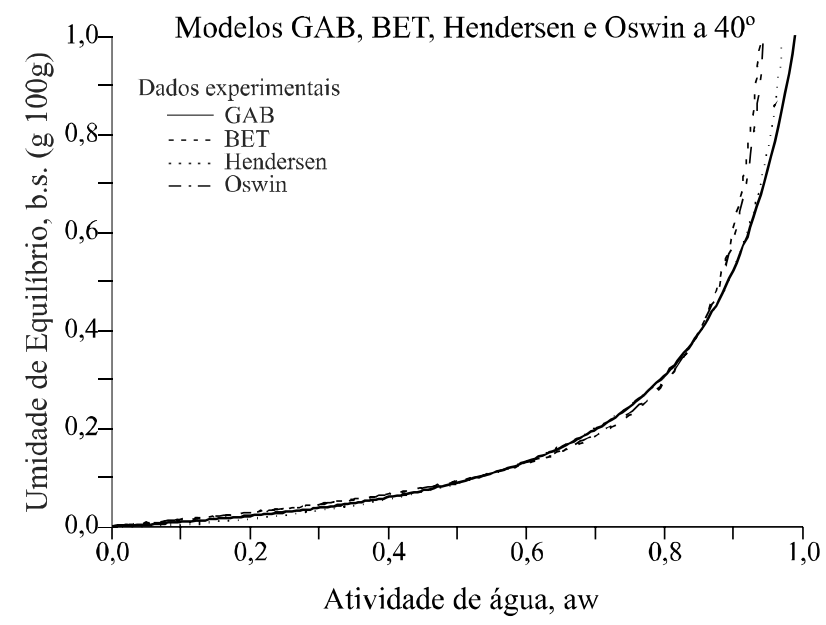

De acordo com ambas as figuras apresentadas verificou-se que para atividades de até 0,6 , o aumento da umidade de equilíbrio foi pequeno, quando comparado às umidades atingidas em atividades de água maiores, o que também foi observado por Righetto e Netto (2005) e por Gabas et al. (2007). Nesta última região, a água exerce uma forte influência sobre a estabilidade do pó, uma vez que, estando na forma de moléculas livres, pode dissolver componentes, resultando em uma aceleração de reações indesejáveis.

Esta construção das isotermas de adsorção dos pós de goiaba atomizado encerrou-se em um período de 8 dias e, foram observadas algumas mudanças 
nas características físicas dos pós armazenados em diferentes umidades relativas, sendo verificado que os pós armazenados sob umidades relativas até $48 \%$ $\left(\mathrm{K}_{2} \mathrm{CO}_{3}\right)$, as partículas permaneceram na forma de pó solto. Já os armazenados sob umidades relativas entre $57(\mathrm{NaBr})$ e $76 \%\left(\mathrm{SnCl}_{2}\right)$ as partículas começaram a apresentar formação de aglomerados que não se soltavam tão facilmente e, os armazenados sob umidades relativas acima de $84 \%(\mathrm{KCl})$ ficaram com a aparência de um aglomerado úmido e pegajoso. Este período de construção das isotermas para os pós de goiaba foi bem inferior ao observado por Bezerra (2009) em seu estudo sobre comportamento higroscópico de pós de diferentes variedades de manga que precisou de 20 dias para que ocorresse este equilíbrio.

Verificou-se na Tabela 2 que os valores do conteúdo de umidade na monocamada molecular $\left(X_{m}\right)$ e da constante relacionada ao calor de sorção da camada molecular (C) das equações de GAB e BET, nas diferentes temperaturas analisadas, são próximos e apresentaram comportamento similares. Oliveira et al. (2009), em seu estudo das isotermas de dessorção da coroa do abacaxi, observaram este mesmo comportamento.

Quanto o valor de C, observou-se que o aumento da temperatura de 25 para $40{ }^{\circ} \mathrm{C}$ provocou também um aumento neste valor, fato que pode estar relacionado às mudanças irreversíveis associadas ao aumento da temperatura como reações enzimáticas e desnaturação de proteínas.

$\mathrm{O}$ parâmetro $\mathrm{K}$ do modelo de $\mathrm{GAB}$ apresentou valores praticamente constantes e em torno de 1 apresentado variações pequenas entre 0,8580 a 0,9151 . E, os valores médios para os parâmetros de "a" e "b", tanto para o modelo de Henderson quanto para o modelo de Oswin, não apresentaram, praticamente, influência da temperatura.

\section{CONCLUSÕES}

1. Todos os parâmetros analisados sofreram alterações após o processo, porém pode-se concluir que o processo de atomização foi adequado para este produto, garantido assim a sua estabilidade por um período mais longo de armazenamento;

2. Em relação às isotermas de adsorção, o modelo Henderson, em todas as temperaturas avaliadas, foi aquele que melhor se ajustou para o pó de goiaba atomizado com erro variando de 09,93 a 12,09\% e coeficiente de correlação variando de 0,9900 a 0,9934 .

\section{AGRADECIMENTOS}

À UFC e INCT pela infra-estrutura disponibilizada para a realização do estudo e ao $\mathrm{CNPq}$ pela bolsa de estudo fornecida a primeira autora.

\section{REFERÊNCIAS}

ADHIKARI, B. et al. Effect of addition of maltodextrin on drying kinetics and stickiness of sugar and acid-rich foods during convective drying: experiments and modelling. Journal of Food Engineering, v. 62, n. 1, p. 53-68, 2004.

AGÊNCIA NACIONAL DE VIGILÂNCIA SANITÁRIA. Resolução da Diretoria Colegiada - RDC n 272, de 22 de setembro de 2005. Dispõe sobre o "Regulamento Técnico para produtos de vegetais, produtos de frutas e cogumelos comestíveis". Diário Oficial da União, Brasília, DF, 2005a.

AGÊNCIA NACIONAL DE VIGILÂNCIA SANITÁRIA. Resolução da Diretoria Colegiada - RDC no 269, de 22 de setembro de 2005. Dispõe sobre o "Regulamento Técnico sobre a ingestão diária recomendada (IDR) de proteína, vitaminas e minerais”. Diário Oficial da União, Brasília, DF, 2005b.

ANSELMO, G. C. S. et al. Determinação da higroscopicidade do cajá em pó por meio da secagem por atomização. Revista de Biologia e Ciências da Terra, v. 6, n. 2, p. 58-65, 2006.

AQUALAB. Analisador de atividade de água Decagon. Brasil: ABRASEQ, 1997. $21 \mathrm{p}$.

AZZOLINI, M. et al. Ripening of "Pedro Sato" guava: study on its climateric or no-climateric nature. Brazilian Journal of Plant Physiology, v. 17, n. 3, p. 299-306, 2005.

BEZERRA, S. T. Comportamento higroscópico de pós de diferentes variedades de manga (Mangifeta indica L.) 2009. 100 f. Dissertação (Mestrado em Tecnologia de Alimentos) Centro de Ciência Agrárias. Universidade Federal do Ceará, Fortaleza, 2009.

BRASIL. Ministério da Agricultura, Pecuária e Abastecimento. Instrução Normativa $\mathrm{n}^{\circ} 1$, de 07 de janeiro de 2000. Dispõe sobre o "Regulamento Técnico Geral para fixação dos Padrões de Identidade e Qualidade para Polpa de Fruta”, Diário Oficial da União, Brasília, DF, 2000.

CALEGUER, V. F.; BENASSI, M. T. Efeito da adição de polpa, carboximetilcelulose e goma arábica nas características sensoriais e aceitação de preparados em pó para refresco sabor laranja. Revista Ciência e Tecnologia de Alimentos, v. 27, n. 2, p. 270-277, 2007.

CANO-CHAUCA, M. et al. Effect of the carriers on the microstructure of mango powder obtained by spray drying and its funcional characterization. Innovative Food Science \& Emerging Technologies, v. 6, n.4, p. 420-428, 2005.

CARVALHO, H. A. et al. Efeito da atmosfera modificada sobre componentes da parede celular da goiaba. Ciência e Agrotecnologia, v. 25, n. 3, p. 605-615, 2001. 
HÉRBRARD, A. et al. Hydration properties of durum wheat semolina: influence of particle size. Powder Technology, v. 130, n. 1/3, p. 211-218, 2003.

INSTITUTO ADOLFO LUTZ. Normas Analíticas, métodos químicos e físicos para análise de alimentos. $3{ }^{a}{ }^{a}$ ed. São Paulo: Instituto Adolfo Lutz, 2004, v. 1.

INSTITUTO BRASILEIRO DE GEOGRAFIA E ESTATÍSTICA. Produção Agrícola Municipal. 2009.

GABAS, A. L. et al. Effect of maltodextrin and arabic gum in water vapor sorption thermodynamic properties of vacuum dried pineapple pulp powder. Journal of Food Engineering, v. 82, n. 2, p. 246-252, 2007.

GREESPAN, L. Humidity fixed points of binary satured aqueous solutions. Journal of Research of the National of Standards A. Physics and Chemistry, v. 81, n. 1, p. 89-96, 1977.

KUROZAWA, L. E.; El-AOUAR; A. A., MUR, F. E. X. Obtenção de isotermas de cogumelo in natura e desidratado osmoticamente. Ciência e Tecnologia de Alimentos, v. 25, n. 4, p. 828-834, 2005.

OLIVEIRA, F. M. N.; FIGUEIRÊDO, R. M. F.; QUEIROZ, A. J. M. Análise comparativa de polpas de pitanga integral, formulação e em pó. Revista Brasileira de Produtos Agroindustriais, v. 8, n. 1, p. 25-33, 2006.

OLIVEIRA, M. M. et al.. Isotermas de dessorção da coroa do abacaxi. Revista Tecnologia \& Ciência Agropecuária, v. 3, n. 1, p. 47-52, 2009.

RIBEIRO, V. G. et al. Armazenamento de goiabas 'Paluma' sob refrigeração e em condição ambiente, com e sem tratamento com cera de carnaúba. Revista Brasileira de Fruticultura, v. 27, n. 2, p. 203-206, 2005.

RIGHETTO, A. M.; NETTO, F. M. Effect of encapsulating materials on water sorption, glass transition and stability of juice from immature acerola. International Journal of Food Properties, v. 8, n. 2, p. 337-346, 2005.

STROHECKER, R.; HENNING, H. M. Analisis de vitaminas: métodos comprobados. Madrid: Paz Montalvo, 1967. 428 p.

VILA, M. T. R. et al. Caracterização química e bioquímica de goiabas armazenadas sob refrigeração e atmosfera modificada. Ciência e Agrotecnologia, v. 31, n. 5, p. 1435-1442, 2007.

WANG, H.; ZHANG, S.; CHEN, G. Glass transition and state diagram for fresh and freeze-dried Chinese gooseberry. Journal of Food Engineering, v. 84, n. 2, p. 307-312, 2008. 